\title{
SEPs and CMEs during cycle 23
}

\author{
Pertti Mäkelä $^{1}$, Nat Gopalswamy ${ }^{2}$, Seiji Yashiro ${ }^{3}$, Sachiko Akiyama ${ }^{1}$, \\ Hong $\mathrm{Xie}^{1}$ and Eino Valtonen ${ }^{4}$ \\ ${ }^{1}$ Dept. of Physics, Catholic University of America, \\ 200 Hannan Hall, Washington, DC 20064, USA \\ email: pertti.makela, sachiko . akiyama, hong. xie@nasa.gov \\ ${ }^{2}$ Nasa Goddard Space Flight Center, \\ Code 695, Greenbelt, MD 20771, USA \\ email: nat.gopalswamy@nasa.gov \\ ${ }^{3}$ Interferometrics, Inc, \\ 13454 Sunrise Valley Drive, Herndon, VA 20171, USA \\ email: seiji.yashiro@nasa.gov \\ ${ }^{4}$ Dept. of Physics \& Astronomy, University of Turku, \\ Vesilinnantie 5, FI-20014 Turku University, Finland \\ email: eino.valtonen@utu.fi
}

\begin{abstract}
We present a study of solar energetic particles (SEPs) in association with coronal mass ejections (CMEs) and type II radio bursts. The particle and CME observations cover the years 1996-2007. We find that heavy-ion events in association with type II bursts and proton events are produced in more western and most energetic CMEs. In addition, the source distribution of type II associated proton events with heavy ions reminds the source distribution expected for events with flare particles. Therefore, the estimation of relative contributions by flares and shocks in SEP events and separation of suggested different particle acceleration models is complicated.
\end{abstract}

Keywords. Acceleration of particles, Sun: coronal mass ejections (CMEs), Sun: particle emission

\section{Introduction}

Large Solar Energetic Particle (SEP) events are closely associated with Coronal Mass Ejections (CMEs). It is believed that solar wind particles are accelerated by CME-driven coronal and interplanetary (IP) shock fronts (Reames 1999). However, based on heavy-ion observations Cane et al. (2003) have suggested that higher-energy SEPs could originate from solar flares. Instead, Tylka et al. (2005) attribute variations in high-energy heavy-ion abundances to different shock geometries and seed particle populations. Coronal and IP shocks may also generate type II radio bursts. Type II bursts occur either continuously or intermittently in metric, decameter-hectometric $(\mathrm{DH})$ and kilometric wavelengths. The emission wavelength depends on the density of the ambient plasma, which decreases with distance from the Sun. The metric type II bursts are emitted by shocks in dense coronal plasma close to the Sun $\left(\lesssim 2.5 \mathrm{R}_{S}\right)$ and kilometric type II bursts by IP shocks in less dense plasma further out from the Sun ( $\left.\gtrsim 10 R_{S}\right)$. Previously Gopalswamy (2003) has found that large SEP events are all associated with DH type II bursts. The association of CMEs, proton events and type II burst has also been studied recently by Gopalswamy et al. (2008). In this study we concentrate on CMEs associated heavy-ion events and DH type II radio bursts. We also use proton events for comparison. 
Table 1. Average properties of CMEs.

\begin{tabular}{lccc}
\hline $\begin{array}{l}\text { Heavy-ion } \\
\text { Enhancement }\end{array}$ & \multicolumn{3}{c}{ CME properties } \\
& Speed $[\mathrm{km} / \mathrm{s}]$ & Width [deg] & Halo-\% \\
\hline Yes & $1518 \pm 160$ & $176 \pm 35$ & 72 \\
No & $1111 \pm 130$ & $166 \pm 28$ & 56 \\
\hline
\end{tabular}

\section{Data analysis}

Our analysis focus on CMEs associated with proton events and DH type II radio bursts. Particle and CME measurements by the Energetic and Relativistic Nuclei and Electron (ERNE; Torsti et al. 1995) instrument and by Large Angle and Spectrometric Coronagraph (LASCO; Brueckner et al. 1995) cover the period 1996-2007. Both instruments are onboard the Solar and Heliospheric Observatory (SOHO) spacecraft. We search for proton intensity increases in the $1.8-4.1 \mathrm{MeV}, 4.1-12.7 \mathrm{MeV}, 13.8-28.0 \mathrm{MeV}$ and 25.9-50.8 MeV energy channels. Plasma measurements by Proton Monitor (Hovestadt et al. 1995), also onboard SOHO, are used to identify events associated with IP shocks, i.e. Energetic Storm Particle (ESP) events, and Corotating Interaction Regions (CIRs). We inspect the $\mathrm{O}$ and Fe intensities in $4-15 \mathrm{MeV} / \mathrm{n}$ and $43-70 \mathrm{MeV} / \mathrm{n}$ energy channels for concurrent heavy-ion enhancements. Proton event onsets are compared with the LASCO CME catalogue (http://cdaw.gsfc.nasa.gov/CME_list/) in order to identify a possible CME. We search for an associated DH type II radio burst observed by Wind/WAVES (Bougeret et al. (1995); http://lep694.gsfc.nasa.gov/waves.waves.html; http://cdaw.gsfc.nasa.gov/CME_list/radio/waves_type2.html) and a solar source location based on a list of GOES X-ray flares (Solar Geophysical Data).

\section{Results and discussion}

In Table 1 we give the average properties with respective statistical errors of CMEs associated with DH type II bursts and proton events with and without a heavy-ion enhancement. CMEs with heavy ions are faster and possibly wider than CMEs without heavy ions. In addition, larger fraction of CMEs with heavy ions is halo CMEs. The properties of CMEs without heavy-ions resemble the general population CMEs with type II bursts studied by Gopalswamy et al. (2005). They found that the average speed of type II associated CMEs is $1115 \mathrm{~km} / \mathrm{s}$, the average width is $139^{\circ}$, and $42.5 \%$ are halo CMEs. CMEs associated with SEPs and type II bursts are wider and faster than an average CME (Gopalswamy et al. 2008). Therefore, it appears that heavy-ion events are produced in the most energetic CMEs.

The distributions of solar sources (flares) of proton events with and without heavyion enhancement are plotted in Fig. 1. Proton events with type II bursts and heavyion events originate from the western hemisphere, mostly near the western limb (left panel). Events without heavy-ion enhancements are evenly distributed over solar longitudes (right panel). Therefore, even in proton events were type II radio emission indicate the existence of a shock, a good connection to the acceleration site in the western hemisphere is essential for the detection of heavy ions at $1 \mathrm{AU}$. The western location suggest that the heavy ions are accelerated near the nose of the shock where it is strongest. Furthermore, the source distribution of type II associated proton events with heavy ions reminds the source distribution expected for SEP events with particles accelerated in flares. Therefore, the estimation of relative contributions by flares and shocks in SEP events is complicated. The two models suggested by Cane et al. (2003) and Tylka et al. (2005) cannot be separated based on source locations alone. 

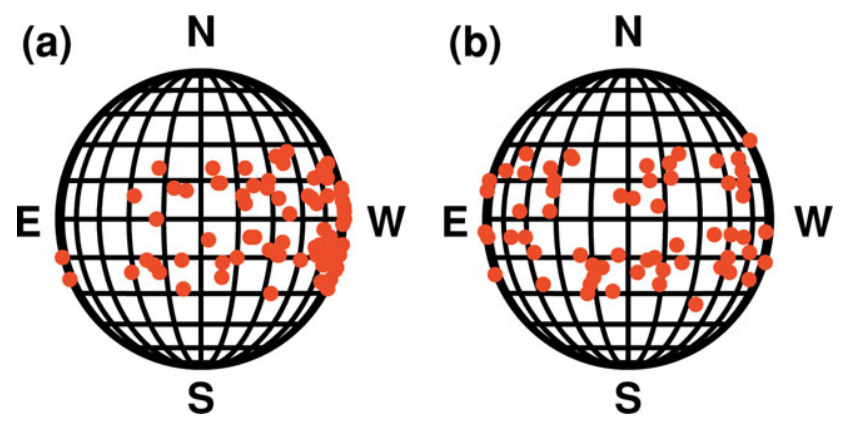

Figure 1. Solar source (flare) locations of type II associated proton events (a) with and (b) without a heavy-ion increase.

\section{Acknowledgements}

SOHO is an international cooperation project between ESA and NASA. This research was supported by NASA grant NNX08AD60A.

\section{References}

Bougeret, J.-L., Kaiser, M. L., Kellogg, P. J., Manning, R., Goetz, K., Monson, S. J., Monge, N., Friel, L., Meetre, C. A., Perche, C., Sitruk, L., \& Hoang, S. 1995, Space Sci. Revs, 71, 231

Brueckner, G. E., Howard, R. A., Koomen, M. J., Korendyke, C. M., Michels, D. J., Moses, J. D., Socker, D. G., Dere, K. P., Lamy, P. L., Llebaria, A., Bout, M. V., Schwenn, R., Simnett, G. M., Bedford, D. K., \& Eyles, C. J. 1995, Solar Phys., 162, 357

Cane, H. V., von Rosenvinge, T. T., Cohen, C. M. S., \& Mewaldt, R. A. 2003 Geophys. Res. Lett., 30, 8017

Gopalswamy, N. 2003, Geophys. Res. Lett., 30, 8013

Gopalswamy, N., Aguilar-Rodriguez, E., Yashiro, S., Nunes, S., Kaiser, M. L., \& Howard, R. A. 2005, J. Geophys. Res., 110, A12S07

Gopalswamy, N., Yashiro, S., Akiyama, S., Mäkelä, P., Xie, H., Kaiser, M. L., Howard, R. A., \& Bougeret, J.-L. 2008, Ann. Geophys., 26, 3033

Hovestadt, D., Hilchenbach, M., Bürgi, A., Klecker, B., Laeverenz, P., Scholer, M., Grünwaldt, H., Axford, W. I., Livi, S., Marsch, E., Wilken, B., Winterhoff, H. P., Ipavich, F. M., Bedini, P., Coplan, M. A.,Galvin, A. B., Gloeckler, G., Bochsler, P., Balsiger, H., Fischer, J., Geiss, J., Kallenbach, R., Wurz, P., Reiche, K.-U., Gliem, F., Judge, D. L., Ogawa, H. S., Hsieh, K. C., Möbius, E., Lee, M. A., Managadze, G. G.,Verigin, M. I., \& Neugebauer, M. 1995, Solar Phys., 162, 441

Reames, D. V. 1999, Space Sci. Revs, 90, 413

Torsti, J., Valtonen, E., Lumme, M., Peltonen, P., Eronen, T., Louhola, M., Riihonen, E., Schultz, G., Teittinen, M., Ahola, K., Holmlund, C., Kelhä, V., Leppälä, K., Ruuska, P., \& Strömmer, E. 1995, Solar Phys., 162, 505

Tylka, A. J., Cohen, C. M. S., Dietrich, W. F., Lee, M. A., Maclennan, C. G., Mewaldt, R. A., Ng, C. K., \& Reames, D. V. 2005, ApJ, 625, 474 